\title{
Sensor Selection and Power Allocation via Maximizing Bayesian Fisher Information for Distributed Vector Estimation
}

\author{
Mojtaba Shirazi, Alireza Sani, and Azadeh Vosoughi \\ Department of Electrical Engineering and Computer Science \\ University of Central Florida \\ Email: mojsh@knights.ucf.edu, Alireza@knights.ucf.edu, azadeh@ucf.edu
}

\begin{abstract}
In this paper we study the problem of distributed estimation of a Gaussian vector with linear observation model in a wireless sensor network (WSN) consisting of $K$ sensors that transmit their modulated quantized observations over orthogonal erroneous wireless channels (subject to fading and noise) to a fusion center, which estimates the unknown vector. Due to limited network transmit power, only a subset of sensors can be active at each task period. Here, we formulate the problem of sensor selection and transmit power allocation that maximizes the trace of Bayesian Fisher Information Matrix (FIM) under network transmit power constraint, and propose three algorithms to solve it. Simulation results demonstarte the superiority of these algorithms compared to the algorithm that uniformly allocates power among all sensors.
\end{abstract}

Index Terms-Distributed estimation, linear observation model, Bayesian Fisher Information Matrix, sensor selection, power allocation, multiple-choice Knapsack problem.

\section{INTRODUCTION}

Researchers have made tremendous contributions to the study of distributed estimation problem for energy constrained sensor networks [1] $-[5]$. Furthermore, significant progress has been made toward data selection approaches and specifically designing distributed sensor selection/activation algorithms to optimize the performance of resource constrained sensor networks [6]-[15]. The authors in [10], [11] studied the sensor selection problem that minimizes parameter estimation error, and described a heuristic algorithm, based on convex optimization, that approximately solves this problem. [11] focused on a general nonlinear observation model and formulated the sensor selection problem as the design of a sparse vector, considering several functions of the Cramér-Rao Bound (CRB) performance measures. [12] introduced a unified framework to jointly design the optimal sensor selection and collaboration schemes subject to a certain information or energy constraint. The authors in [13], [14] considered the problem of sensor selection for parameter estimation under a network transmit power constraint. In particular, [13] considered a linear observation model with correlated measurement noises and sought optimal sensor activation algorithm by formulating an optimization problem, in which the trace of Fisher Information Matrix (FIM) is maximized subject to energy constraints. [14] considered estimation of a function of a random parameter, and analyzed the effect of measurement noise variance on the optimal power allocation, for different network topologies. The authors in [15] developed a multi-tier distributed computing infrastructure for sensor selection and data allocation using mobile device cloud, where all tasks are executed in parallel by sharing the workload among multiple nearby sensors. In our previous works [16] [17], we presented our preliminary results on deriving Bayesian CRB matrix and studied the behavior of its trace, with respect to the system parameters. In our recent work [18], considering the problem of distributed estimation of a Gaussian random vector with linear observation model, we derived the Bayesian FIM. We studied two transmit power optimization problems that maximize the trace of FIM and log-determinant of FIM under network transmit power constraint, and demonstrated that the estimation performance significantly enhances, using the transmit power allocation solutions corresponding to these two problems, compared with uniform power allocation among all sensors. Furthermore, a comparison was made between the estimation performance of coherent and noncoherent reception at the fusion center (FC).

Adopting the same system model as in [18], in this paper we study the sensor selection and power allocation problem that maximizes the trace of Bayesian FIM under network transmit power constraint. We propose three algorithms to address this problem, and compare the performances of these algorithms in simulation results.

\section{System Model and Problem Statement}

We consider a network of $K$ sensors, where each sensor observes a common zero-mean Gaussian vector $\boldsymbol{\theta}=$ $\left[\theta_{1}, \theta_{2}, \ldots, \theta_{q}\right]^{T} \in \mathbb{R}^{q}$ with covariance matrix $\mathcal{C}_{\boldsymbol{\theta}}=\mathbb{E}\left\{\boldsymbol{\theta} \boldsymbol{\theta}^{T}\right\}$. We assume a linear observation model for sensor $k$ as:

$$
x_{k}=\mathbf{a}_{k}^{T} \boldsymbol{\theta}+n_{k}, \quad k=1, \ldots, K
$$

where $\mathbf{a}_{k}=\left[a_{k_{1}}, a_{k_{2}}, \ldots, a_{k_{q}}\right]^{T} \in \mathbb{R}^{q}$ is known observation gain vector and $n_{k}$ denotes zero-mean Gaussian observation noise with variance $\sigma_{n_{k}}^{2}$. We assume that $n_{k}$ 's are uncorrelated observation noises across sensors and also are uncorrelated with $\boldsymbol{\theta}$. Sensor $k$ employs a uniform scalar quantizer with $M_{k}=2^{L_{k}}$ quantization levels $m_{k, l}=\frac{\left(2 l-1-M_{k}\right) \Delta_{k}}{2}$ for $l=1, \ldots, M_{k}$, where $\Delta_{k}$ denotes quantization step size and index $l$ indicates the quantization level $m_{k, l}$. We assume $p\left(\left|x_{k}\right| \geq \tau_{k}\right) \approx 0$ for some $\tau_{k}$ value. Hence, we choose 


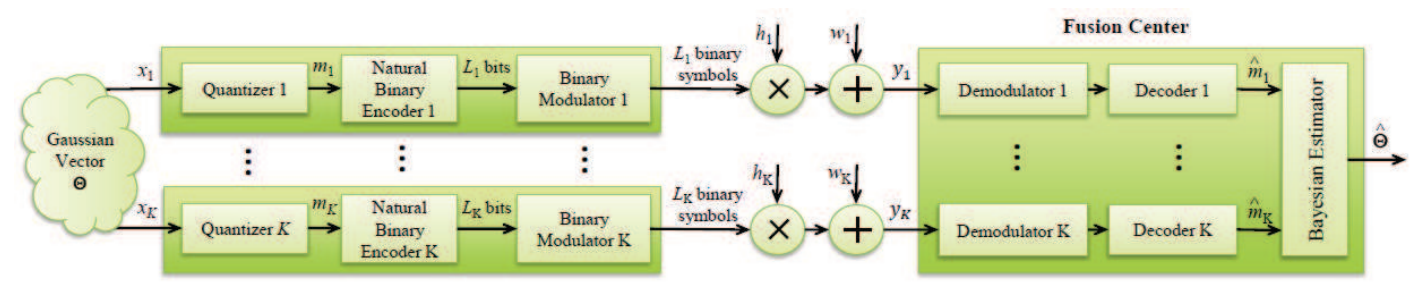

Fig. 1: Our system model consists of $K$ sensors and a FC, that is tasked with estimating a Gaussian vector $\boldsymbol{\theta}$, via fusing collective received signals.

$\Delta_{k}=\frac{2 \tau_{k}}{\left(2^{L}-1\right)}$ [1], [5]. The quantizer maps $x_{k}$ to one of the quantization levels $m_{k} \in\left\{m_{k, 1}, \ldots, m_{k, M_{k}}\right\}$. Following quantization, sensor $k$ employs a fixed length encoder, which encodes the index $l$ corresponding to the quantization level $m_{k, l}$ to a binary sequence of length $L_{k}=\log _{2} M_{k}$ according to natural binary encoding [1], [5], and finally modulates these $L_{k}$ bits into $L_{k}$ binary symbols. Let $P_{k}$ denote transmit power corresponding to $L_{k}$ symbols from sensor $k$, which is equally distributed among $L_{k}$ symbols. Each sensor employs a Binary Phase Shift Keying (BPSK) modulator, which maps each bit of $L_{k}$-bit sequence into one symbol with transmit power $P_{k} / L_{k}$.

Sensors send their modulated symbols to the FC over orthogonal flat fading channels, with complex-valued fading coefficient $h_{k}$. Suppose channel $h_{k}$ remains constant during the transmission of $L_{k}$ symbols. Denote $\nu_{k, i}$ as communication channel noise during the transmission of $i$-th symbol of $L_{k}$ symbols corresponding to sensor $k$. We assume $\nu_{k, i}$ 's are independent and identically distributed across $L_{k}$ transmitted symbols and $K$ channels, $\nu_{k, i} \sim \mathcal{C N}\left(0,2 \sigma_{\nu_{k}}^{2}\right)$. Furthermore, there is a constraint on the network transmit power, i.e., $\sum_{k=1}^{K} P_{k} \leq P_{t o t}$, and thus, only a subset of sensors might be active at each task period.

Let $\hat{m}_{k}$ denote the recovered quantization level corresponding to sensor $k$, where in general, $\hat{m}_{k} \neq m_{k}$ due to communication channel errors. The FC processes channel output corresponding to sensor $k$ to recover transmitted quantization level $\hat{m}_{k} \in\left\{\hat{m}_{k, 1}, \ldots, \hat{m}_{k, M_{k}}\right\}$. Having $\left\{\hat{m}_{1}, \ldots, \hat{m}_{K}\right\}$, the FC applies a Bayesian estimator to form the estimate $\hat{\boldsymbol{\theta}}$. Under certain regularity conditions that are satidfied by Gaussian vectors, one can derive the $q \times q$ FIM, denoted here as $\boldsymbol{J}$. We refer the interested readers to section IV of [18] for the derivation of matrix $\boldsymbol{J}$. From (30) in [18], we obtain:

$$
\begin{aligned}
\operatorname{tr}(\boldsymbol{J}) & =\operatorname{tr}\left(\mathcal{C}_{\boldsymbol{\theta}}{ }^{-1}\right)+\sum_{k=1}^{K} t_{k}\left(P_{k}\right), \\
t_{k}\left(P_{k}\right) & =\frac{\mathbf{a}_{k}^{T} \mathbf{a}_{k}}{2 \pi \sigma_{n_{k}}^{2}} \mathbb{E}_{\boldsymbol{\theta}}\left\{G_{k}\left(\boldsymbol{\theta}, P_{k}\right)\right\} \\
G_{k}\left(\boldsymbol{\theta}, P_{k}\right) & =\sum_{t=1}^{M_{k}} \frac{\left(\sum_{l=1}^{M_{k}} \alpha_{k, t, l}\left(P_{k}\right) \dot{\beta}_{k, l}(\boldsymbol{\theta})\right)^{2}}{\sum_{l=1}^{M_{k}} \alpha_{k, t, l}\left(P_{k}\right) \beta_{k, l}(\boldsymbol{\theta})} .
\end{aligned}
$$

Note that $t_{k}$ is a function of $P_{k}$. Due to the cap on the network transmit power, only a subset of the sensors might be active at each task period. So we introduce a sensor selection parameter $w_{k} \in\{0,1\}$, to indicate whether or not sensor $k$ is selected to participate in the distributed estimation task and transmit to the FC. Our goal is to study sensor selection and transmit power allocation that maximizes $\operatorname{tr}(\boldsymbol{J})$, subject to network transmit power constraint. In other words, we are interested in solving the following constrained optimization problem:

$$
\begin{aligned}
\underset{P_{k}, w_{k}, \forall k}{\operatorname{maximize}} & \sum_{k=1}^{K} w_{k} t_{k}\left(P_{k}\right) \\
\text { s.t. } & \sum_{k=1}^{K} P_{k} \leq P_{t o t}, P_{k} \in \mathbb{R}^{+}, \forall k \\
& w_{k} \in\{0,1\}, \forall k
\end{aligned}
$$

\section{Constrained Maximization of BAyesian Fisher INFORMATION}

The constrained optimization problem in (P1) is a nonconvex mixed integer non-linear programming problem which is NP-hard combinatorial problem and its computational complexity increases exponentially with the problem size.

In the following, we propose three different algorithms to tackle (P1). In the first two algorithms, we use two different relaxations of $(\overline{\mathrm{P} 1})$ and in the third algorithm, we propose a reformulation of $(\overline{\mathrm{P} 1})$ and solve it. As part of the first algorithm, relaxation (P2) is obtained based on uniform transmit power allocation among all sensors and relaxation of the Boolean constraints in $(\overline{\mathrm{P} 1})$. Problem $(\overline{\mathrm{P} 2})$ becomes a linear programing problem and we solve it using simplex method. We refer to the first algorithm as uniform-select-uniform (USU) algorithm. In the second algorithm, we iteratively select a new sensor in a greedy manner until a stopping criteria is met. As part of the second algorithm, relaxation $(\mathrm{P} 3)$ is obtained when we assume $\left\{w_{k}\right\}_{k=1}^{K}$ is given. Problem ( $\mathrm{P} 3$ becomes a convex problem and we find the solution using Newton's method. We refer to the second algorithm as greedy algorithm. The third formulation ( $\mathrm{P} 4 \mathrm{f}$ is obtained based on discretizing transmit power and is called in the literature a multiple-choice knapsack problem (MCKP). Although ( $\mathrm{P} 4)$ is an NP-hard problem, we can find the solution in pseudo-polynomial time using dynamic programming. We refer to the third algorithm as MCKP algorithm. Define $\boldsymbol{t}=\left[t_{1}, \ldots, t_{K}\right]^{T}$, and let $\boldsymbol{P}=\left[P_{1}, \ldots, P_{K}\right]^{T}$ and $\boldsymbol{w}=\left[w_{1}, \ldots, w_{K}\right]^{T}$ be the vectors of sensors' powers and sensor selection parameters, respectively. Suppose $\boldsymbol{P}^{*}$ and $\boldsymbol{w}^{*}$ are final solutions for the algorithms.

- USU Algorithm: Initialize with $i=1$. Emplying uniform power allocation among all sensors, $\boldsymbol{P}$ and $\boldsymbol{t}$ can be obtained. Then, we find the best set of active sensors $S_{i+1}$ with cardinality $\left|S_{i+1}\right|=i$ via solving the following Boolean relaxed 


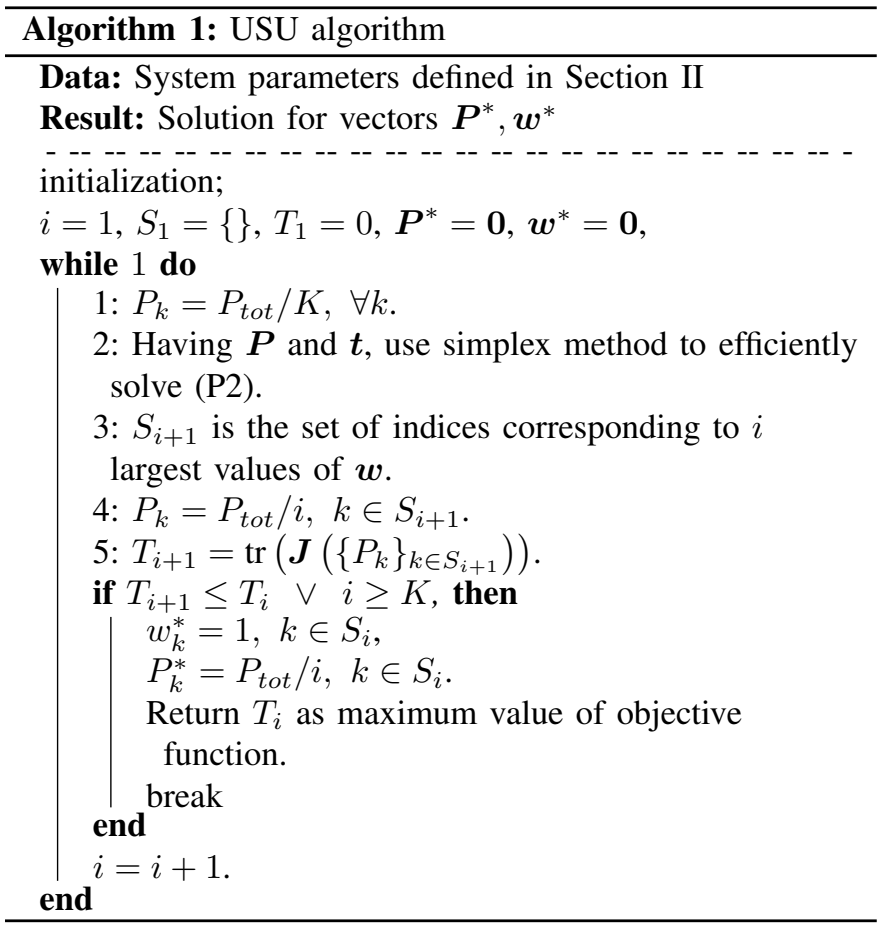

sensor selection problem:

$$
\begin{aligned}
\underset{\boldsymbol{w}}{\operatorname{maximize}} & \boldsymbol{w}^{T} \boldsymbol{t} \\
\text { s.t. } & \mathbf{1}^{T} \boldsymbol{w} \leq i, \\
& \boldsymbol{w} \in[0,1]^{K} .
\end{aligned}
$$

Given the set $S_{i+1}$ we uniformly allocate the power among the selected $i$ sensors, i.e., $P_{k}=P_{t o t} / i, k \in S_{i+1}$ and repeat this procedure while incrementing $i$ until a stopping criteria is met. A summary is described in Algorithm 1 .

- Greedy Algorithm: Define $\mathcal{A}$ and $\mathcal{I}$ as sets of active and inactive sensors, respectively. Initialize with $i=1, \mathcal{A}=\{\}$, $\mathcal{I}=\{1, \ldots, K\}$. For each sensor in the inactive set $\mathcal{I}$, say $\mathcal{I}_{j}, j=1, \ldots,|\mathcal{I}|$, use Newton's method to find the optimal solution $\left\{P_{k}^{\prime}\right\}_{k \in \mathcal{U}_{j}}$ for the following power allocation problem:

$$
\begin{aligned}
\underset{P_{k}, k \in \mathcal{U}_{j}}{\operatorname{maximize}} & \mathbf{1}^{T} \boldsymbol{t} \\
\text { s.t. } & \sum_{k \in \mathcal{U}_{j}} P_{k} \leq P_{\text {tot }}, P_{k} \in \mathbb{R}^{+}, \forall k \in \mathcal{U}_{j},
\end{aligned}
$$

where $\mathcal{U}_{j}=\mathcal{A} \cup \mathcal{I}_{j}$, and save $Y_{j}=\operatorname{tr}\left(J\left(\left\{P_{k}^{\prime}\right\}_{k \in \mathcal{U}_{j}}\right)\right)$. In [18], we argue that $(\overline{\mathrm{P} 3})$ is a convex programming problem. Then we select the sensor $\mathcal{I}_{j}$ which gives the largest performance improvement, i.e. the one corresponding to $\max \left\{Y_{1}, \ldots, Y_{|\mathcal{I}|}\right\}$. We repeat this procedure while updating $\mathcal{A}$ and $\mathcal{I}$ and incrementing $i$ until a stopping criteria is met. A summary is described in Algorithm 2.

- MCKP Algorithm: We discretize $P_{t o t}$ into $N$ samples $\boldsymbol{P}_{d}=$

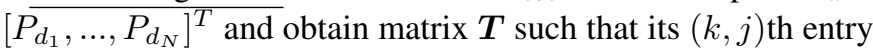
$[\boldsymbol{T}]_{k, j}=t_{k}\left(P_{d_{j}}\right), \quad k=1, \ldots, K, j=1, \ldots, N$. Let $\boldsymbol{W}$ be a $K \times N$ matrix whose entries can be zeros or ones. Then (P1)

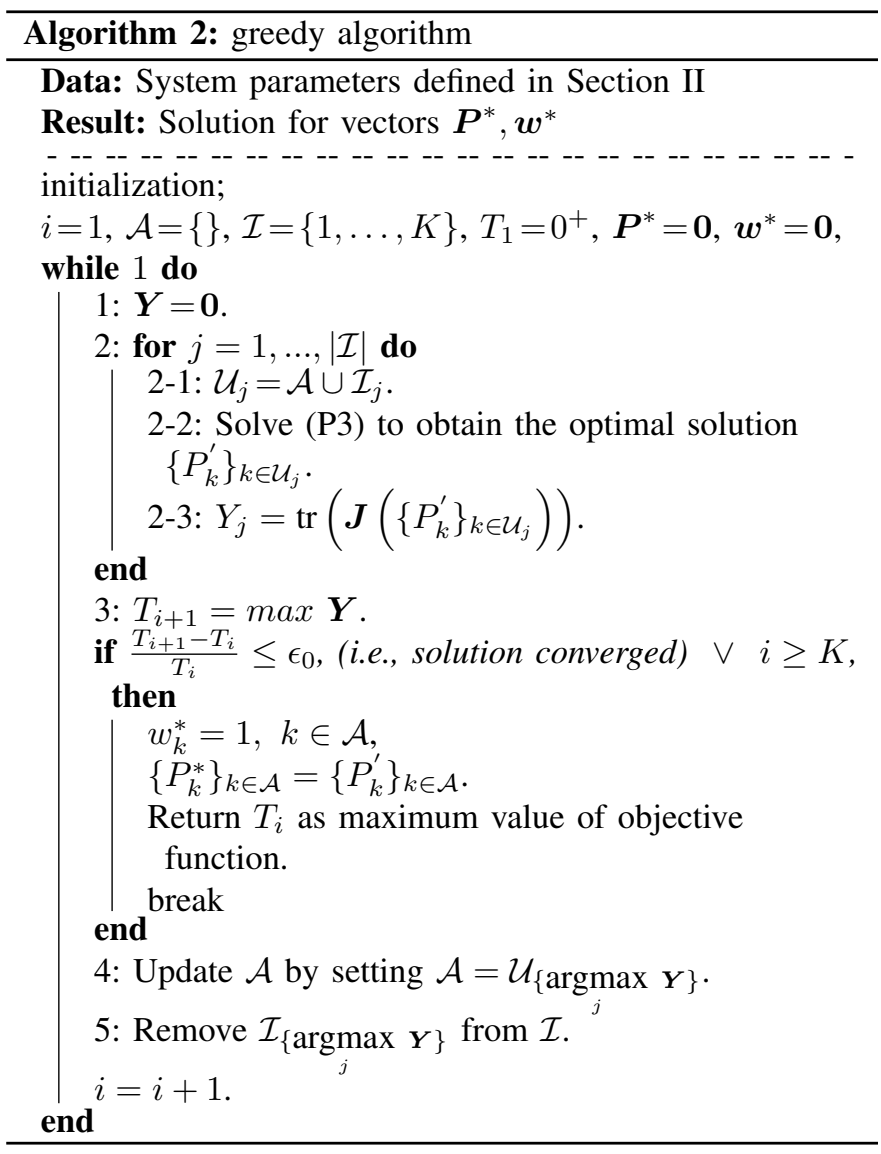

becomes:

$$
\begin{aligned}
\underset{\boldsymbol{W}}{\operatorname{maximize}} & \mathbf{1}^{T} \underbrace{(\boldsymbol{W} \circ \boldsymbol{T})}_{\text {Hadamard product }} \mathbf{1} \\
\text { s.t. } & \mathbf{1}^{T}\left(\boldsymbol{W} \circ\left(\mathbf{1} \boldsymbol{P}_{d}^{T}\right)\right) \mathbf{1} \leq P_{\text {tot }}, \\
& {[\boldsymbol{W}]_{k} \mathbf{1}=1, k=1, \ldots, K, } \\
& \boldsymbol{W} \in\{0,1\}^{K \times N},
\end{aligned}
$$

in which $[\boldsymbol{W}]_{k}$ is $k$-th row of $\boldsymbol{W}$. The second constraint states that only one value from vector $\boldsymbol{P}_{d}$ should be chosen for each sensor, which in fact is the associated power of that sensor. Problem (P4) is a MCKP that is NP-hard, but it can be solved in pseudo-polynomial time through dynamic programming [19].

\section{Numerical AND Simulation Results}

We assume a zero mean Gaussian vector $\boldsymbol{\theta}=\left[\theta_{1}, \theta_{2}\right]^{T}$ with $\mathcal{C}_{\boldsymbol{\theta}}=[4,0.5 ; 0.5,0.25]$, and $\sigma_{n_{k}}=1, \sigma_{\nu_{k}}=1,\left|h_{k}\right|=0.7, L_{k}=$ 3 for all $k$. We choose a proper $\tau_{k}$ based on the observation model and joint pdf of the unknown vector such that $p\left(\left|x_{k}\right| \geq\right.$ $\left.\tau_{k}\right) \approx 0$. To this end, we choose $\tau_{k}=3 \sqrt{\sigma_{n_{k}}^{2}+\mathbf{a}_{k}^{T} \mathcal{C}_{\boldsymbol{\theta}} \mathbf{a}_{k}}$.

First, we consider a homogeneous sensor network with $\mathbf{a}_{k}=$ $[0.6,0.8]^{T}$ for all $k$. Fig. 2 a shows that an increase in $P_{t o t}$ results in increase in $\operatorname{tr}(\boldsymbol{J})$. Moreover, we are interested to know the optimal number of sensors that maximizes $\operatorname{tr}(\boldsymbol{J})$. 
From Fig. $2 \mathrm{~b}$ we observe that for a given $P_{t o t}$, it is always beneficial to uniformly allocate $P_{t o t}$ among all of the sensors.

Next, we consider $K=20$ sensors that are randomly deployed in a $2 m \times 2 m$ field. Assuming Cartesian coordinate system with origin at center of the field, the goal is to estimate two signal sources $\boldsymbol{\theta}=\left[\theta_{1}, \theta_{2}\right]^{T}$. The distance between $\theta_{i}$ located at $\left(x_{t_{i}}, y_{t_{i}}\right)$ and sensor $k$ located at $\left(x_{s_{k}}, y_{s_{k}}\right)$ is:

$d_{k i}=\sqrt{\left(x_{s_{k}}-x_{t_{i}}\right)^{2}+\left(y_{s_{k}}-y_{t_{i}}\right)^{2}}, \quad k=1, \ldots, 20, \quad i=1,2$

Let $d_{0 i}$ be distance of $\theta_{i}$ from origin, $d_{01}=d_{02}=1 \mathrm{~m}$, and $\mathbf{a}_{k}^{T}=\left[\left(\frac{d_{01}}{d_{k 1}}\right)^{n},\left(\frac{d_{02}}{d_{k 2}}\right)^{n}\right]$ where $n$ is signal decay exponent which is approximately 2 for distances $\leq 1 \mathrm{~km}$ [20]. Fig. 3 compares the performance of 3 algorithms USU, greedy, and MCKP. We also consider uniform power allocation among all sensors, i.e., $\left\{P_{k}=P_{t o t} / K\right\}_{k=1}^{K}$ where no sensor selection is carried out. We refer to this algorithm as UFA algorithm. For MCKP, our simulations suggest that $N=100$ samples are sufficient to reach the optimal solution. Fig. 3a illustrates that greedy and MCKP algorithms perform similarly, and they both outperform USU algorithm, which is intuitive. However, the performance of USU algorithm is not far from other algorithms, which highlights the advantage of sensor selection even when power is uniformly allocated among sensors. Moreover, all three algorithms significantly outperform UFA algorithm. Fig. 3b reveals that USU algorithm always selects less number of sensors compared to other algorithms, which can save energy in battery-powered sensor networks.

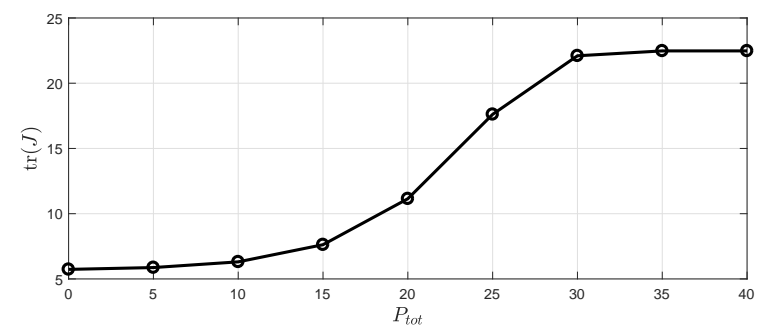

(a)

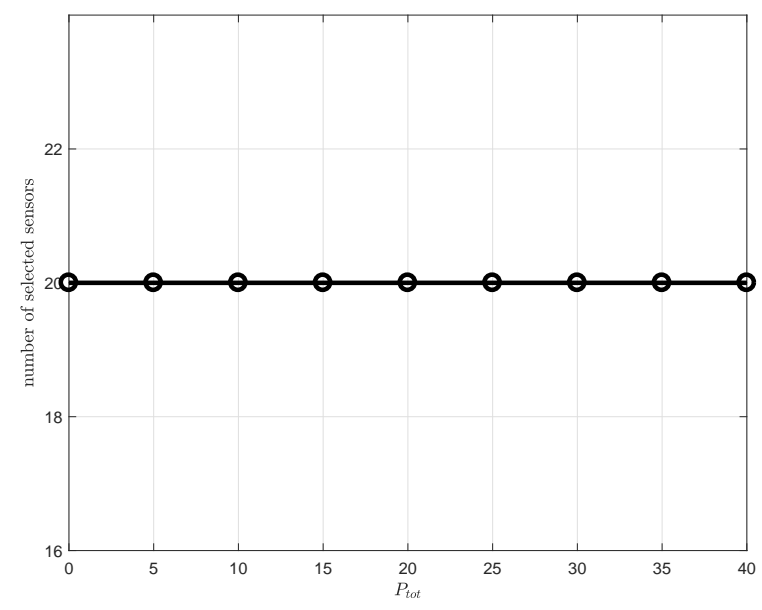

(b)

Fig. 2: Homogeneous sensor network: (a) $\operatorname{tr}(\boldsymbol{J})$, and (b) number of selected sensors, versus $P_{\text {tot }}$.

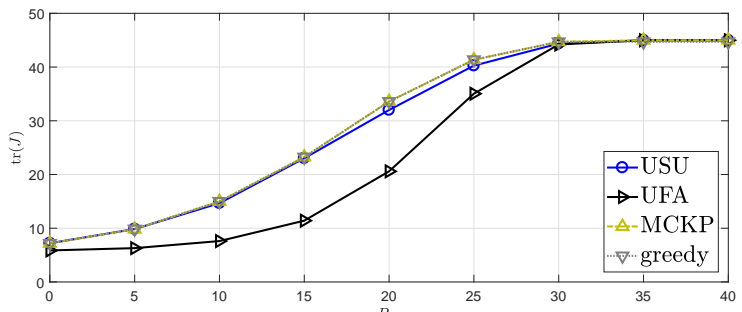

(a)

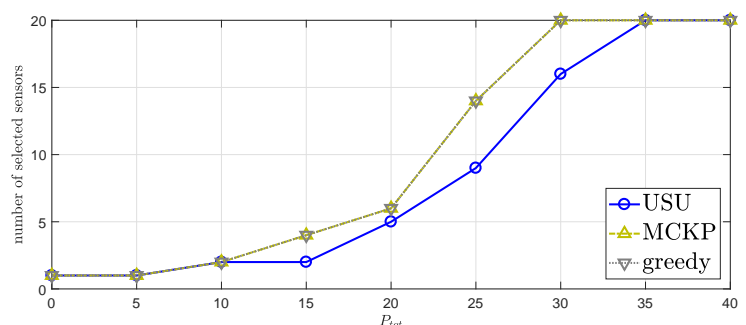

(b)

Fig. 3: Heterogeneous sensor network: comparison of (a) $\operatorname{tr}(\boldsymbol{J})$, and (b) number of selected sensors, versus $P_{t o t}$ for different algorithms.

\section{CONCLUSIONS}

We considered the problem of sensor selection and power allocation to maximize the trace of Bayesian Fisher information matrix $\boldsymbol{J}$ under network transmit power constraint for distributed estimation of a zero mean Gaussian vector in wireless sensor networks. Three algorithms named as USU, greedy, and MCKP algorithms were proposed and their performances were compared numerically. Simulation results demonstarted the superiority of these algorithms compared to the algorithm that allocates power equally among all sensors. Moreover, the advantage of USU algorithm is that its performance is close to those of other algorithms and also, less number of sensors get activated compared to other algorithms, which contributes to energy saving in battery-powered sensor networks.

\section{ACKNOWLEDGMENT}

This research is supported by NSF under grants CCF1341966 and CCF-1319770.

\section{REFERENCES}

[1] A. Sani and A. Vosoughi, "On distributed vector estimation for power and bandwidth constrained wireless sensor networks," IEEE Transactions on Signal Processing, vol. PP, no. 99, pp. 1-1, 2016.

[2] J.-J. Xiao, S. Cui, Z.-Q. Luo, and A. Goldsmith, "Power scheduling of universal decentralized estimation in sensor networks," Signal Processing, IEEE Transactions on, vol. 54, no. 2, pp. 413-422, Feb 2006.

[3] J. Li and G. AlRegib, "Distributed estimation in energy-constrained wireless sensor networks," IEEE Transactions on Signal Processing, vol. 57, no. 10, pp. 3746-3758, Oct 2009.

[4] X. Luo and G. Giannakis, "Energy-constrained optimal quantization for wireless sensor networks,", EURASIP J. Adv. Signal Process., p. 12, 2008.

[5] M. Chaudhary and L. Vandendorpe, "Power constrained linear estimation in wireless sensor networks with correlated data and digital modulation," Signal Processing, IEEE Transactions on, vol. 60, no. 2, pp. 570-584, Feb 2012.

[6] M. J. Moghaddam, M. Hosseini, and R. Safabakhsh, "Traffic light control based on fuzzy q-leaming," in 2015 The International Symposium on Artificial Intelligence and Signal Processing (AISP), 2015, pp. 124128 
[7] M. Hosseini, M. A. Salehi, and R. Gottumukkala, "Enabling interactive video streaming for public safety monitoring through batch scheduling," in 2017 IEEE 19th International Conference on High Performance Computing and Communications; IEEE 15th International Conference on Smart City; IEEE 3rd International Conference on Data Science and Systems (HPCC/SmartCity/DSS), 2017, pp. 474-481.

[8] M. Sedghi, M. Georgiopoulos, and G. C. Anagnostopoulos, "Sparse inductive embedding: An explorative data visualization technique," in 2017 IEEE 29th International Conference on Tools with Artificial Intelligence (ICTAI), Nov 2017, pp. 618-622.

[9] S. Vahidian, S. Assa, and S. Hatamnia, "Relay selection for securityconstrained cooperative communication in the presence of eavesdropper's overhearing and interference," IEEE Wireless Communications Letters, vol. 4, no. 6, pp. 577-580, Dec 2015.

[10] S. Joshi and S. Boyd, "Sensor selection via convex optimization," IEEE Transactions on Signal Processing, vol. 57, no. 2, pp. 451-462, Feb 2009.

[11] H. Jamali-Rad, A. Simonetto, X. Ma, and G. Leus, "Distributed sparsityaware sensor selection," IEEE Transactions on Signal Processing, vol. 63, no. 22, pp. 5951-5964, Nov 2015.

[12] S. Liu, S. Kar, M. Fardad, and P. K. Varshney, "Sparsity-aware sensor collaboration for linear coherent estimation," IEEE Transactions on Signal Processing, vol. 63, no. 10, pp. 2582-2596, May 2015.

[13] S. Liu, S. Chepuri, M. Fardad, E. Masazade, G. Leus, and P. Varshney, "Sensor selection for estimation with correlated measurement noise," IEEE Transactions on Signal Processing, vol. PP, no. 99, pp. 1-1, 2016.

[14] G. Thatte and U. Mitra, "Sensor selection and power allocation for distributed estimation in sensor networks: Beyond the star topology," IEEE Transactions on Signal Processing, vol. 56, no. 7, pp. 2649-2661, July 2008.

[15] M. P. Hosseini, D. Pompili, K. Elisevich, and H. Soltanian-Zadeh, "Optimized deep learning for EEG big data and seizure prediction $\mathrm{BCI}$ via internet of things," IEEE Transactions on Big Data, vol. PP, no. 99, 2017.

[16] M. Shirazi and A. Vosoughi, "Bayesian cramer-rao bound for distributed vector estimation with linear observation model," in 2014 IEEE 25th Annual International Symposium on Personal, Indoor, and Mobile Radio Communication (PIMRC), Sept 2014, pp. 712-716.

[17] _ "Bayesian cramer-rao bound for distributed estimation of correlated data with non-linear observation model," in 2014 48th Asilomar Conference on Signals, Systems and Computers, Nov 2014, pp. 1484 1488.

[18] _ "Fisher information maximization for distributed vector estimation in wireless sensor networks," CoRR, 2017. [Online]. Available: http://arxiv.org/abs/1705.00803

[19] D. Pisinger, "A minimal algorithm for the multiple-choice knapsack problem," European Journal of Operational Research, vol. 83, no. 2, pp. $394-410,1995$.

[20] Y. Hu and D. Li, "Energy-based collaborative source localization using acoustic micro-sensor array," EURASIP J. Appl. Signal Process., vol. 2003, no. 4, pp. 321-337, March 2003. 